\title{
18F-fluoride PET/MR in cardiac amyloid: A comparison study with aortic stenosis and age- and sex-matched controls
}

\author{
Jack P. M. Andrews, MD, ${ }^{\mathrm{a}}$ Maria Giovanni Trivieri, $\mathrm{MD}, \mathrm{PhD},{ }^{\mathrm{b}, \mathrm{c}}$ \\ Russell Everett, MD, PhD, ${ }^{a}$ Nicholas Spath, MD, ${ }^{a}$ Gillian MacNaught, PhD, ${ }^{\mathrm{d}}$ \\ Alastair J. Moss, MD, PhD, ${ }^{a}$ Mhairi K. Doris, MD, ${ }^{a}$ Tania Pawade, MD, PhD, ${ }^{a}$ \\ Edwin J. R. van Beek, MD, PhD, ${ }^{\mathrm{a}, \mathrm{d}}$ Christophe Lucatelli, PhD, ${ }^{\mathrm{d}}$ \\ David E. Newby, MD, PhD, ${ }^{\text {a }}$ Philip Robson, PhD, ${ }^{\text {b,c }}$ Zahi A. Fayad, MD, PhD, , c \\ and Marc R. Dweck, MD, PhD ${ }^{a}$ \\ a British Heart Foundation Centre for Cardiovascular Science, University of Edinburgh, Edinburgh, \\ UK \\ b Icahn School of Medicine at Mount Sinai, New York, NY \\ c BioMedical Engineering and Imaging Institute, New York, NY \\ d Edinburgh Imaging, Queen's Medical Research Institute University of Edinburgh, Edinburgh, UK
}

Received May 25, 2020; accepted Aug 19, 2020

doi: $10.1007 / \mathrm{s} 12350-020-02356-1$

Objectives. Cardiac MR is widely used to diagnose cardiac amyloid, but cannot differentiate AL and ATTR subtypes: an important distinction given their differing treatments and prognoses. We used PET/MR imaging to quantify myocardial uptake of $18 \mathrm{~F}$-fluoride in ATTR and AL amyloid patients, as well as participants with aortic stenosis and age/sex-matched controls.

Methods. In this prospective multicenter study, patients were recruited in Edinburgh and New York and underwent 18F-fluoride PET/MR imaging. Standardized volumes of interest were drawn in the septum and areas of late gadolinium enhancement to derive myocardial

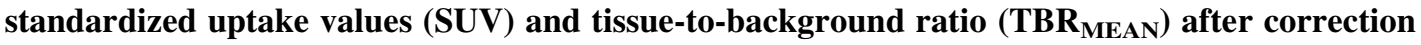
for blood pool activity in the right atrium.

Results. 53 patients were scanned: 18 with cardiac amyloid (10 ATTR and 8 AL), 13 controls, and 22 with aortic stenosis. No differences in myocardial TBR values were observed between participants scanned in Edinburgh and New York. Mean myocardial TBR MEAN $_{\text {values }}$ in ATTR amyloid $(1.13 \pm 0.16)$ were higher than controls $(0.84 \pm 0.11, P=.0006)$, aortic stenosis $(0.73 \pm 0.12, P<.0001)$, and those with AL amyloid $(0.96 \pm 0.08, P=.01)$. TBR MEAN $_{\text {values }}$

Electronic supplementary material The online version of this article (https://doi.org/10.1007/s12350-020-02356-1) contains supplementary material, which is available to authorized users.

The authors of this article have provided a PowerPoint file, available for download at SpringerLink, which summarizes the contents of the paper and is free for re-use at meetings and presentations. Search for the article DOI on SpringerLink.com.

The authors have also provided an audio summary of the article, which is available to download as ESM, or to listen to via the JNC/ASNC Podcast.

Funding JPMA is supported by BHF Clinical Research Training Fellowship no. FS/17/51/33096. MGT is supported by the National Institutes of Health grant 5T32HL007824-18 and KL2 TR001435. PMR and ZAF are supported by National Institutes of Health grant (R01 HL071021). DEN (CH/09/002, RE/18/5/34216) is supported by the British Heart Foundation and a Wellcome Trust Senior Investigator Award (WT103782AIA). MRD is supported by the Sir Jules Thorn Biomedical Research Award 2015 (15/JTA) and by the British Heart Foundation (FS/14/78/31020). The Edinburgh Imaging is supported by the National Health Service Research Scotland (NRS) through National Health Service Lothian Health Board.

Reprint requests: Jack P. M. Andrews, MD, British Heart Foundation Centre for Cardiovascular Science, University of Edinburgh, Room SU.305, Chancellor's building, 51 Little France Crescent, EdinburghEH164SB; jack.andrews@ed.ac.uk

$1071-3581 / \$ 34.00$

Copyright (C) 2020 The Author(s) 
within areas of late gadolinium enhancement provided discrimination between patients with ATTR $(1.36 \pm 0.23)$ and all other groups (e.g., AL $[1.06 \pm 0.07, P=.003])$. A TBR $_{\text {MEAN }}$ threshold $>1.14$ in areas of LGE demonstrated $100 \%$ sensitivity (CI 72.25 to $100 \%$ ) and $100 \%$ specificity (CI 67.56 to $100 \%$ ) for ATTR compared to AL amyloid (AUC $1, P=.0004$ ).

Conclusion. Quantitative 18F-fluoride PET/MR imaging can distinguish ATTR amyloid from other similar phenotypes and holds promise in improving the diagnosis of this condition. (J Nucl Cardiol 2022;29:741-9.)

Key Words: PET $\cdot$ PET/MR $\cdot$ 18F-fluoride $\cdot$ Amyloid $\cdot$ Aortic stenosis $\cdot$ CMR

\begin{tabular}{|c|c|}
\hline \multicolumn{2}{|c|}{ Abbreviations } \\
\hline AL & Amyloid light chain \\
\hline ATTR & Amyloid transthyretin \\
\hline CMR & Cardiac magnetic resonance \\
\hline SPECT & $\begin{array}{l}\text { Single photon emission computerized } \\
\text { tomography }\end{array}$ \\
\hline${ }^{99 \mathrm{~m}} \mathrm{Tc}-$ & ${ }^{99}$ Technicium- diphosphono- \\
\hline DPD & propanedicarboxylic acid \\
\hline${ }^{99 \mathrm{~m}} \mathrm{Tc}-$ & hydroxymethylene \\
\hline HMDP & diphosphonate \\
\hline${ }^{99 \mathrm{~m}} \mathrm{Tc}-$ & ${ }^{99}$ Technicium- pyrophosphate \\
\hline PYP & \\
\hline PET/MR & $\begin{array}{l}\text { Positron emission tomography/mag- } \\
\text { netic resonance }\end{array}$ \\
\hline MGUS & $\begin{array}{l}\text { Monoclonal gammopathy of unknown } \\
\text { significance }\end{array}$ \\
\hline CMRA & $\begin{array}{l}\text { Cardiac magnetic } \\
\text { angiography }\end{array}$ \\
\hline GRE & Gradient recalled echo \\
\hline PET & Positron emission tomography \\
\hline OSEM & Ordered subsets maximization \\
\hline $\mathrm{MBq}$ & Megabecquerels \\
\hline VOI & Volume of interest \\
\hline SUV $_{\text {MEAN }}$ & Mean standardized uptake value \\
\hline $\mathrm{SUV}_{\text {MAX }}$ & Max standardized uptake value \\
\hline TBR $_{\text {MEAN }}$ & Mean target to background ratio \\
\hline $\mathrm{TBR}_{\mathrm{MAX}}$ & Max target to background ratio \\
\hline LGE & Late gadolinium enhancement \\
\hline ANOVA & Analysis of variance \\
\hline ROC & Receiver operating characteristic \\
\hline AUC & Area under the curve \\
\hline TAVI & Transfemoral aortic valve insertion \\
\hline
\end{tabular}

See related editorial, pp. $750-752$

\section{INTRODUCTION}

Systemic amyloidosis represents a spectrum of conditions characterized by disordered protein folding and fibrils formation. The two predominant forms affecting the heart are amyloid light chain (AL) and amyloidosis transthyretin (ATTR; hereditary or wild- type). ${ }^{1}$ Differentiation between these two forms is important because they are associated with very different prognoses but most importantly different treatment strategies. $^{2} \mathrm{AL}$ amyloid is associated with a poor outcome but may be amenable to treatment with chemotherapy. ${ }^{3}$ In contrast, ATTR amyloid is associated with a better prognosis and is potentially responsive to novel therapies that have been developed to reduce TTR protein aggregation. ${ }^{4}$

The diagnosis of cardiac amyloidosis is challenging. Echocardiography is frequently unable to differentiate amyloid from other forms of left ventricular hypertrophy such as that from aortic stenosis. Furthermore, some studies have suggested that cardiac amyloidosis is present in $6 \%$ to $29 \%$ of patients with significant aortic stenosis. ${ }^{5-7}$ Other data have also indicated that patients with aortic stenosis and concurrent cardiac amyloidosis have an adverse prognosis despite aortic valve replacement. It is, therefore, important to identify aortic stenosis patients with co-existent amyloidosis both in terms of predicting prognosis and because it influences interventional treatment decisions.

Endomyocardial biopsy is still the gold standard to make a diagnosis of cardiac amyloid but it is invasive, may be prone to sampling error, and is associated with procedure-related risk. Alongside histology, cardiovascular magnetic resonance (CMR) is increasingly being used to aid in the diagnosis of amyloidosis based largely upon the characteristic pattern of late gadolinium enhancement and T1 mapping ${ }^{8-11}$ : CMR alone cannot, however, reliably distinguish AL from ATTR subtypes. SPECT bone tracers $\left({ }^{99 \mathrm{~m}} \mathrm{Tc}-\mathrm{DPD},{ }^{99 \mathrm{~m}} \mathrm{Tc}-\mathrm{HMDP}\right.$ and ${ }^{99 \mathrm{~m}}$ Tc-PYP) can provide this discrimination, demonstrating increased cardiac uptake in ATTR compared to $\mathrm{AL}$ amyloid and control subjects, but these approaches only provide a semi-quantitative assessment of activity. ${ }^{12,13}$ Conversely, we have shown in prior work that 18F-fluoride, a PET bone tracer, ${ }^{14,15}$ might have the potential to discriminate between AL and TTR amyloid in a quantitative manner, and could allow improved discrimination and monitoring of response to therapy. ${ }^{16,17}$ Lastly, the simultaneous acquisition of $18 \mathrm{~F}$ fluoride-PET and MR imaging using a single co-registered scan (PET/MR) has the advantage of combining 
the two modalities in the assessment of patients with amyloid.

In this novel prospective multicenter study, we aim to build on the initial findings by Trivieri et al, ${ }^{17}$ and investigate whether 18F-fluoride PET/MR imaging can help with the diagnosis of TTR amyloidosis and differentiate patients with AL cardiac amyloid, aortic stenosis, and age/sex-matched controls.

\section{METHODS}

\section{Patient Recruitment}

This multicenter hybrid imaging study was conducted between December 2015 and June 2018 at two sites: The British Heart Foundation centre for Cardiovascular Science at the University of Edinburgh, UK and the Icahn School of Medicine in New York, USA. All participants were older than 50 years. Patients with cardiac amyloid were recruited from outpatient clinics and inpatient wards over both sites. The diagnosis of AL or TTR amyloid was established on histological analysis of biopsy samples in all patients bar one who had Multiple Myeloma. This patient had CMR features of amyloid (included in the AL group) but did not have a tissue biopsy. Age/sex-matched subjects were recruited as a negative control group as were patients with aortic stenosis (peak aortic jet velocity of $>2.5 \mathrm{~m} / \mathrm{s}$, with no clinical suspicion of amyloid).

Exclusion criteria for all cohorts included inability to receive iodinated contrast, renal impairment (estimated glomerular filtration rate $\leq 30 \mathrm{~mL} / \mathrm{min} / 1.73 \mathrm{~m}^{2}$ ) or women of child-bearing potential.

For those recruited in Edinburgh, the study was approved by the Scottish Research Ethics Committee and the United Kingdom (UK) Administration of Radiation Substances Advisory Committee. It was performed in accordance with the Declaration of Helsinki and all patients provided written informed consent prior to any study procedures. Subjects recruited in New York had ethical and Institutional Review Board approval for the study (GCO\#01-1032). All participants provided written informed consent. The study was registered on Clinicaltrials.gov (NCT03626584). We did not directly include patient and public involvement (PPI) in this study, but the patient information sheet used in the study was developed with PPI and was reviewed by a committee that includes patient representatives.

\section{Imaging Protocols}

\footnotetext{
${ }^{18}$ F-fluoride positron emission tomography and coronary magnetic resonance angiography This was a multicenter PET/MR study, but where possible protocols were standardized between centers with all patients undergoing simultaneous PET and MR imaging using the same hybrid PET/MR system (Biograph mMR, Siemens Healthcare GmbH, Erlangen, Germany). The MR protocol at each site was the same including long axis cine imaging (3-chamber, 2-chamber, 4-chamber), a short axis cine stack ( $8 \mathrm{~mm}$ thickness, $1.6 \mathrm{~mm}$ gap) coronary magnetic
}

resonance angiography (CMRA for accurate co-registration) performed with $0.2 \mathrm{mmol} / \mathrm{kg}$ of intravenous gadobutrol contrast (Gadovist, Bayer Pharma AG, Germany) and late gadolinium enhancement imaging 10 to 15 minute postcontrast administration. Importantly, the same radial gradient recalled echo (GRE, Siemens work-in-progress \#793F) sequences was acquired for MR attenuation correction at both sites as previously described, ${ }^{6}$. PET imaging was performed 60 to 120 minutes post administration of $125-350 \mathrm{MBq} 18 \mathrm{~F}-$ fluoride. List mode PET data were then reconstructed using e7tools (Siemens Healthcare) applying the radial GRE sequence (2 tissue classes: background [air and lung] and soft tissue [soft tissue and fat]). ${ }^{18,19}$ An Ordered Subsets Expectation Maximization (OSEM) algorithm with the following parameters was employed: $256 \times 256$ matrix, 4 iterations, 21 subsets, $5 \mathrm{~mm}$ Gaussian filter in Edinburgh and $344 \times 344$ matrix, 6 iterations, 21 subsets, $2 \mathrm{~mm}$ Gaussian filter in New York.

PET/MR image analysis All PET/MR images were analyzed at the University of Edinburgh Core Lab by two expert readers (JA, MRD). Accurate co-registration was achieved by aligning ${ }^{18} \mathrm{~F}$-fluoride activity in the blood pool and ascending aorta with the corresponding anatomical structures on the CMRA. ${ }^{20}$ Qualitative and semi-quantitative analysis of the PET images was performed using FusionQuant software (Cedars-Sinai Medical Center, Los Angeles). Radiotracer uptake was analyzed using a standardized protocol (supplemental data). For myocardial analysis two approaches were employed. First, based on previous research showing amyloid infiltration and septal hypertrophy in $79 \%$ of ATTR cases we sampled septal uptake. ${ }^{21}$ This was calculated using cylinders of $3 \mathrm{~mm}$ radius and $15 \mathrm{~mm}$ length set within the septum at mid cavity level on the co-registered co-axial image to generate volumes of interest (VOI, Figure 1, supplementary protocol). Standardized uptake values $\left(\mathrm{SUV}_{\text {MEAN }}\right.$ and $\left.\mathrm{SUV}_{\mathrm{MAX}}\right)$ were calculated for these septal VOIs and corrected for blood pool activity (measured in the right atrium) ${ }^{22}$ to provide tissue-tobackground ratio (TBR $\mathrm{TEAN}_{\mathrm{N}}$ and $\left.\mathrm{TBR}_{\mathrm{MAX}}\right)$. Second, in patients with cardiac amyloid ${ }^{\mathrm{I}} \mathrm{F}$-fluoride uptake was assessed in areas of myocardial LGE. Equal sized volumes of interest (VOI) were placed within areas of LGE demonstrating the greatest visual uptake on short axis slices with SUV $_{\text {MEAN }}$ and

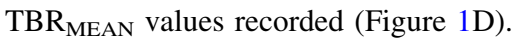

Throughout the study TBR values were used in preference for comparison. In principle the correction of tissue uptake for blood pool measurements made on the same scan adjusts for potential differences in PET values acquired at different scanners. In order to test this principle, we compared

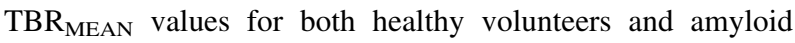
patients imaged in Edinburgh to the equivalent subjects imaged in New York.

\section{Statistical Analysis}

All statistical analyses were performed using GraphPad Prism Version 8.0. A two-sided $P<0.05$ was considered statistically significant. The distribution of all continuous variables was assessed using the Shapiro-Wilk test, which were 
presented using mean \pm standard deviation or median [interquartile range]. Comparisons between groups were performed using the two-sample $t$ test, Mann-Whitney test or ordinary one-way ANOVA as appropriate. Receiver operating characteristic (ROC) curves were generated to determine area under curve (AUC) and perform sensitivity and specificity analyses. We presented all categorical variables as percentages.

\section{RESULTS}

53 patients (33 in Edinburgh and 20 in New York) were scanned and completed the study protocol without complication: 13 controls, 18 with cardiac amyloid (10 ATTR and $8 \mathrm{AL}$ ), and 22 aortic stenosis (Table 1).

Despite differences in scanning protocol and PET reconstruction between the two sites, there was no significant difference in myocardial $\mathrm{TBR}_{\text {MEAN values in }}$ either the control subject nor the amyloid patients (Table 2).

\section{Septal Myocardial 18F-Fluoride Activity}

CMR was normal in all controls (Table 1). Myocardial 18F-fluoride uptake in their normal myocardium was lower than blood pool (septal TBR MEAN $0.86 \pm 0.10$ $P<.0001$ ), allowing the cavity of the blood pool in the left and right ventricles to be delineated and facilitating accurate co-registration of the MR angiogram and PET datasets in 3 dimensions. Patients with aortic stenosis had left ventricular hypertrophy with elevated LV mass index $\left(100 \pm 26 \mathrm{~g} / \mathrm{m}^{2}\right)$ but normal ejection fraction $(69 \pm$ $17 \%)$. 18F-flouride uptake was again lower than blood pool $\left(\mathrm{TBR}_{\text {MEAN }} 0.73 \pm 0.12, P=.03\right)$.

18 patients with cardiac amyloidosis were scanned: $10(56 \%)$ with ATTR and $8(44 \%)$ with AL cardiac amyloid. Mean myocardial mass was elevated at $101 \pm$ $37 \mathrm{~g} / \mathrm{m}^{2}$ with mean ejection fraction of $60 \pm 18 \%$. $15 / 18$ $(83 \%)$ patients had a pattern of diffuse generalized pattern of LGE on CMR (8 with ATTR and 7 with AL). Two patients (1 TTR and 1 AL) had focal LGE, while 1 (TTR) had no appreciable LGE on CMR.

Increased septal $18 \mathrm{~F}$-fluoride activity was observed in patients with ATTR amyloid $(1.13 \pm 0.16)$ compared to healthy volunteers $(0.86 \pm 0.10, P=.0002)$ and patients with both AL amyloid $(0.95 \pm 0.08, P=.01)$ and aortic stenosis $(0.73 \pm 0.12, P<.0001$; Table 3 , Figure $3 \mathrm{~A})$. There was no increase in septal $18 \mathrm{~F}$-fluoride uptake in patients with $\mathrm{AL}$ amyloidosis compared to controls $(0.95 \pm 0.08, P=.54)$.

\section{Myocardial 18F-Fluoride Activity in Areas of Late Gadolinium Enhancement}

In the ATTR patients the most intense myocardial 18F-fluoride uptake was observed in areas of LGE (Figure 2). Indeed, TBR values were 35\% higher in these LGE regions compared to other areas of 'normal' myocardium in the same patients $(1.39 \pm 0.23$ vs $0.90 \pm$ $0.23, P<.0002$ ). Moreover $18 \mathrm{~F}$-fluoride TBR values in areas of LGE provided excellent discrimination between patients with TTR and AL amyloid (Figure 3B) with a TBR $_{\text {MEAN }}$ threshold $>1.14$ providing $100 \%$ sensitivity (CI 72.25 to $100 \%$ ) and $100 \%$ specificity (CI 67.56 to $100 \%$ ) for TTR amyloid (AUC $1, P=.0004$ ).

\section{DISCUSSION}

In the first multicenter study of its kind, we have used hybrid PET/MR to investigate myocardial $18 \mathrm{~F}$ fluoride activity in patients with TTR amyloid, demonstrating increased uptake compared to age- and sexmatched controls and patients with both aortic stenosis and AL amyloid (Table 3 + Figure 3A). Indeed, when sampled within areas of myocardial disease, a TBR MEAN $_{1}$ threshold of 1.14 provided perfect discrimination between patients with ATTR and these other conditions. Our findings are in keeping with our own and recently published work by other groups. ${ }^{17,23,24}$

The diagnosis and treatment of cardiac amyloidosis has advanced markedly over the last 10 years. AL amyloid can now be treated with disease modifying novel chemotherapeutic agents ${ }^{25,26}$, while contemporary TTR therapies target suppression of TTR expression and stabilization of transthyretin, improving patients outcomes. $^{4}$ The accurate diagnosis of TTR amyloid is, therefore, of major importance. However, the mechanism of benefit of these drugs on the myocardium is not well understood and importantly improvements in myocardial performance after therapy are frequently absent. Thus, there is a need for novel imaging techniques that can elucidate the effects of these novel drugs on the myocardium and that can track disease progression and response to therapy. Each of the currently available imaging techniques has well recognized limitations in these regards. CMR is the reference standard imaging test for the diagnosis of cardiac amyloid, but cannot differentiate between AL and TTR amyloid. SPECT bone tracers demonstrate increased uptake in TTR amyloid but provide only imperfect discrimination from $\mathrm{AL}$ and semi-quantitative measurements poorly suited to detecting treatment response. While PET imaging with amyloid specific tracer offers fully quantitative assessments of amyloid burden, these tracers are 


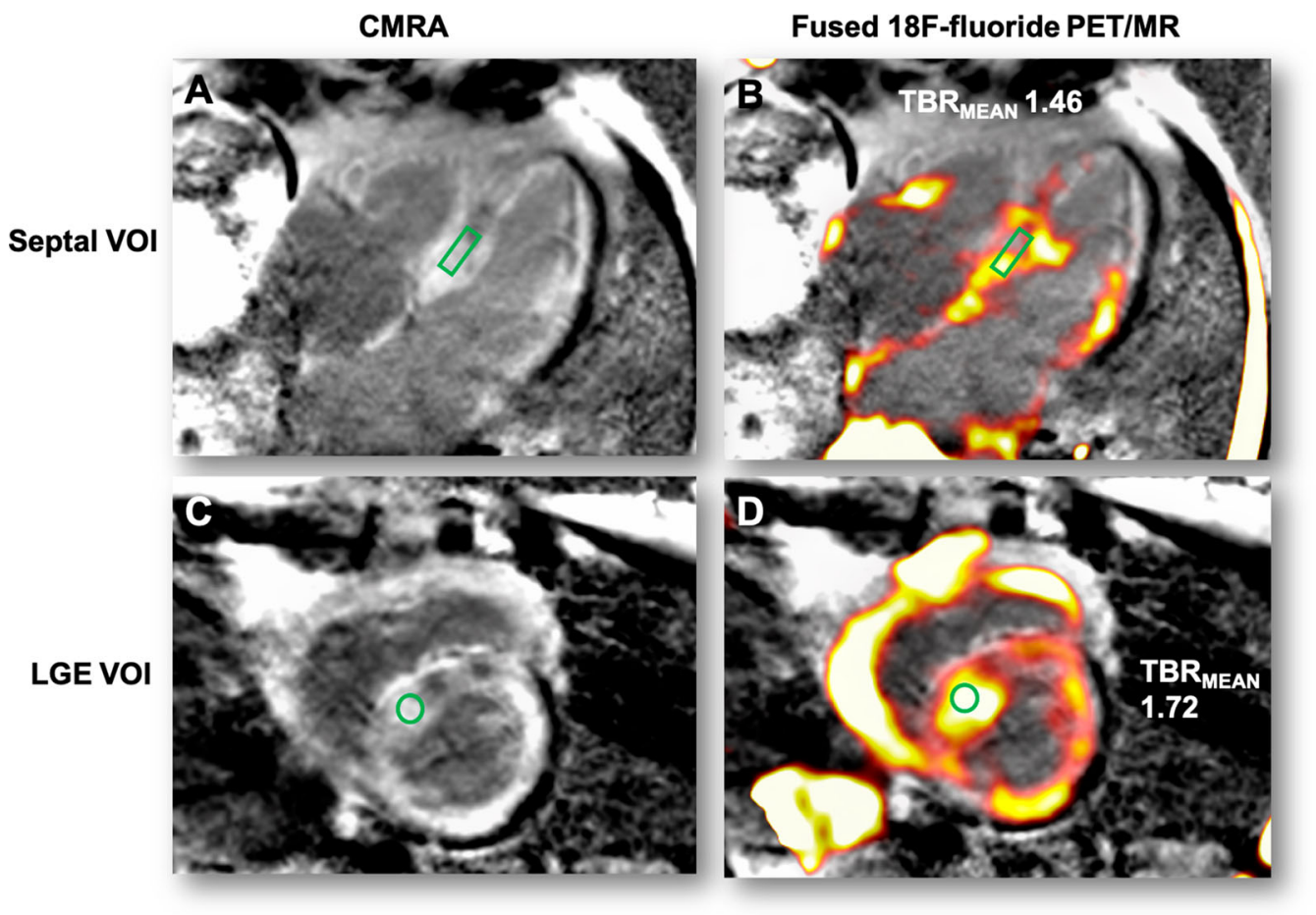

Figure 1. Derivation of myocardial and LGE volumes of interest. Panel $\mathbf{A}$ is a 4-chamber view with LGE showing typical myocardial nulling difficulties in a patient with TTR cardiac amyloidosis. The standardized $3 \times 15 \mathrm{~mm}$ green cylindrical VOI is placed in the septum at the midventricular level. Panel B shows the fused PET/MR with high uptake in the septal and lateral wall

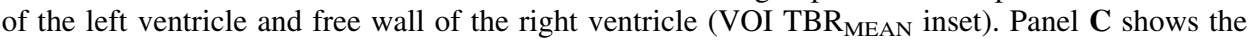
LGE image in the short axis with extensive diffuse LGE affecting most of the myocardium with the corresponding short axis view of the septal VOI. Panel D shows the fused PET/MR image of the same slice with diffuse uptake within the left and right ventricular myocardium. Note the intensely high uptake within the most diseased myocardial segment (within the septal VOI) and the difference in $\mathrm{TBR}_{\mathrm{MEAN}}$ compared to panel $\mathbf{B}$.

expensive and do not fully differentiate between $\mathrm{AL}$ and TTR amyloid.

18F-fluoride PET/MR appears to provide a solution to some of these problems and, therefore, holds promise in the diagnosis and monitoring of patients with TTR amyloid. This approach combines the accurate diagnosis of cardiac amyloid on the CMR with clear discrimination of biopsy proven AL and TTR amyloid on the 18Ffluoride PET in a single scan. Hybrid imaging also facilitates accurate co-registration and comparison of the CMR and PET images. This is an important advantage as it allows regions of interest to be accurately drawn in specific regions of the myocardium including areas of late gadolinium enhancement wherein TBR values provide the greatest discriminatory information. The value of hybrid imaging will be further explored in the upcoming 'I-CARE' study which will evaluate the incremental benefit of tissue imaging with CMR to $18 \mathrm{~F}$ fluoride PET/CT. Finally, although theoretical at present, the fully quantitative nature of PET could potentially provide an assessment of TTR burden that could be used to track disease progression and/or treatment responses to the array of new therapies being developed for this condition. Indeed, PET imaging has been used in a similar fashion in other contexts assessing treatments responses to both atherosclerosis and aortic stenosis (SALTIRE 2 clinicaltrials.gov, NCT02132026).

While patterns of late gadolinium enhancement tend to differ between patients with ATTR and aortic stenosis, we have also demonstrated that $18 \mathrm{~F}$-fluoride PET/MR can too provide excellent discrimination between ATTR and aortic stenosis. This too is becoming an increasingly important and challenging clinical distinction. Recent studies have suggested that up to $12 \%$ patients with significant aortic stenosis undergoing surgical AVR have co-existent cardiac amyloid., ${ }^{5,6}$ Furthermore, $16 \%$ of patients referred for TAVI (transcatheter aortic valve insertion) had uptake (Tc-99m-PYP) consistent with ATTR, 62\% of whom met the criteria for low-flow, low-gradient severe aortic 
Table 1. Participant clinical, radiological, and CMR characteristics

\section{Clinical characteristics}

\begin{tabular}{|c|c|c|c|}
\hline Age & $65 \pm 15$ & $76 \pm 8$ & $70 \pm 9$ \\
\hline Male & $8 / 13(62 \%)$ & $15 / 22(68 \%)$ & $14 / 18(78 \%)$ \\
\hline BMI $\left(\mathrm{kg} / \mathrm{m}^{2}\right)$ & $26.0 \pm 3.0$ & $28.6 \pm 4.2$ & $24.4 \pm 5.1$ \\
\hline Smoking (ex or current) & $3 / 13(23 \%)$ & $9 / 22(41 \%)$ & $8 / 18(44 \%)$ \\
\hline Hypertension & $4 / 13(31 \%)$ & $13 / 22(59 \%)$ & $11 / 18(61 \%)$ \\
\hline Hyperlipidaemia & $4 / 13(31 \%)$ & $11 / 22(50 \%)$ & $8 / 18(44 \%)$ \\
\hline Diabetes & $1 / 13(8 \%)$ & $4 / 22(18 \%)$ & $5 / 18(28 \%)$ \\
\hline Previous myocardial Infarction & $0 / 13(0 \%)$ & $2 / 22(9 \%)$ & $2 / 18(11 \%)$ \\
\hline Previous PCI & $0 / 13(0 \%)$ & $3 / 22(14 \%)$ & $3 / 18(17 \%)$ \\
\hline Imaged in Edinburgh & 6 & 22 & 5 \\
\hline $\begin{array}{l}\text { Administered dose }{ }^{18} \mathrm{~F} \text { - } \\
\text { Fluoride }(\mathrm{MBq})\end{array}$ & $308 \pm 80$ & $201 \pm 58$ & $333 \pm 91$ \\
\hline $\begin{array}{l}\text { PET/MR injection-to-scan } \\
\text { interval (mins) }\end{array}$ & $50 \pm 18$ & $80 \pm 31$ & $55 \pm 22$ \\
\hline $\begin{array}{l}\text { Body surface } \\
\text { area }\left(\mathrm{m}^{2}\right)\end{array}$ & $1.91 \pm 0.20$ & $1.96 \pm 0.17$ & $1.86 \pm 0.26$ \\
\hline LVEDi & $72 \pm 20$ & $76 \pm 24$ & $72 \pm 21$ \\
\hline LVESVi & $18 \pm 5$ & $26 \pm 23$ & $31 \pm 25$ \\
\hline LVSVi & $55 \pm 17$ & $52 \pm 11$ & $40 \pm 11$ \\
\hline Ejection Fraction (\%) & $75 \pm 6$ & $70 \pm 17$ & $60 \pm 18$ \\
\hline LVMi & $64 \pm 8$ & $100 \pm 26$ & $101 \pm 36$ \\
\hline
\end{tabular}

$B M I$, body mass index; $P C I$, percutaneous coronary intervention; $P E T / M R$, positron emission tomography/magnetic resonance; $C M R$, cardiac magnetic resonance; $L V E D i$, left ventricular end diastolic volume indexed; $L V E S V i$, left ventricular end systolic volume indexed; $L V S V i$, left ventricular stroke volume indexed; $L V M i$, left ventricular mass indexed

Table 2. Comparison of $T B R_{M E A N}$ values between centers

\begin{tabular}{|c|c|c|c|}
\hline & Edinburgh TBR $_{\text {MEAN }}$ & New York TBR $_{\text {MEAN }}$ & $P$ value \\
\hline Age/sex-matched controls & $0.84 \pm 0.08$ & $0.88 \pm 0.11$ & $P=.53$ \\
\hline Amyloid & $1.11 \pm 0.21$ & $1.03 \pm 0.04$ & $P=.37$ \\
\hline
\end{tabular}

$T B R$, tissue-to-background ratio

stenosis. ${ }^{27}$ Treatments for aortic stenosis and amyloid differ; ${ }^{3,4}$ in particular patients with ATTR may not be best served with transcutaneous or surgical aortic valve replacement.

Our observations in the healthy control group are also of importance. A key advantage of $18 \mathrm{~F}$-fluoride imaging in the heart is the low myocardial activity, with uptake values $80 \%$ of that observed in the blood pool. This has several implications that facilitate the wider assessment of 18F-fluoride PET as a marker of disease activity in the coronary arteries and heart valves. ${ }^{18,28-31}$ First it allows accurate co-registration of the blood pool signal on the PET with the cardiac chambers on MR angiography. Second it means that even relatively low 18-fluoride uptake in the coronary arteries can be differentiated from blood pool and the activity in the adjacent myocardium. 
Table 3. Comparison of $\mathrm{TBR}_{\mathrm{MEAN}}$ between groups

\begin{tabular}{lccc}
\hline & Septal TBR & LGE & P value \\
\hline Age/sex-matched controls & $0.86 \pm 0.10$ & - & - \\
Aortic stenosis & $0.73 \pm 0.12$ & - & - \\
AL amyloid & $0.96 \pm 0.08$ & $1.06 \pm 0.06$ & $P=.01$ \\
ATTR amyloid & $1.13 \pm 0.16$ & $1.39 \pm 0.23$ & $P=.02$ \\
\hline
\end{tabular}

TBR, tissue-to-background ratio; $L G E$, late gadolinium enhancement; $A L$, amyloid light chain; $T T R$, transthyretin

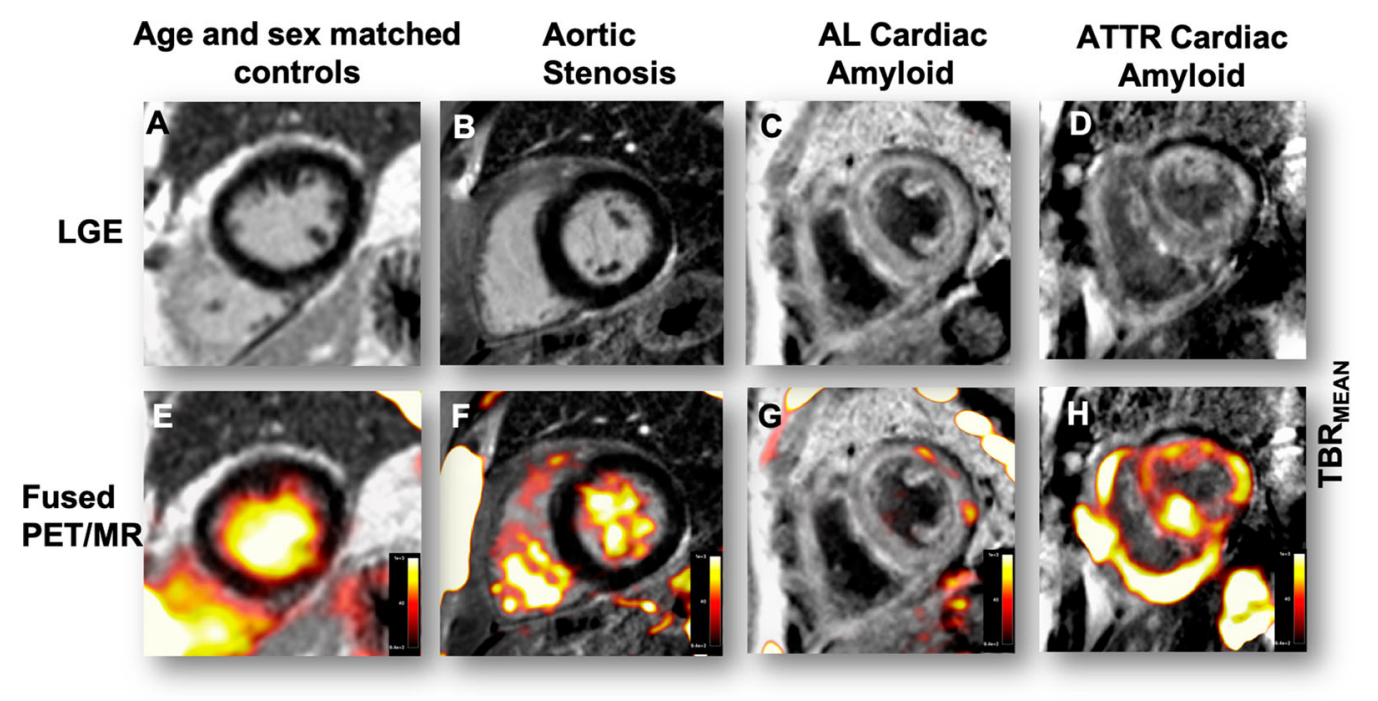

Figure 2. Patterns of 18F-fluoride uptake between cohorts. Columns represent each cohort and rows imaging modality in the short axis view. Panel A shows a delayed enhanced image of a control subject with normal myocardial mass and no LGE. The corresponding fused PET/MR image (E) shows uptake only in the blood pool. Panel $\mathbf{B}$ is a patient with aortic stenosis and elevated LV mass. Note the absence of myocardial $18 \mathrm{~F}$-fluoride uptake on panel $\mathbf{F}$ and similar to the healthy control, uptake is greater in the blood pool than myocardium. Panel $\mathbf{C}$ shows a patient with AL amyloid displaying the characteristic myocardial nulling difficulties with LGE found in cardiac amyloidosis. Panel G shows patchy lateral wall uptake greater than the blood pool. Panel D shows similar LGE findings, but this time in TTR amyloid. Note the striking and extensive biventricular uptake in panel $\mathbf{H}$, much greater than the blood pool and what was seen in AL.

\section{LIMITATIONS}

To our knowledge, this is the largest multicenter study investigating myocardial $18 \mathrm{~F}$-fluoride uptake in cardiac amyloid and while there is an important need for multicenter cardiovascular PET studies, this approach comes with inherent challenges and limitations. These include variation in the injected activity of $18 \mathrm{~F}$-fluoride, reconstruction parameters, and between scanner differences in uptake measurement values. Despite these potential problems we have demonstrated that TBR values appear to correct for many of these between center variations with no difference in TBR values measured in equivalent patients imaged at the two centers. Future multicenter cardiovascular PET studies should, therefore, be encouraged in the knowledge that many between center differences can be overcome.

\section{CONCLUSION}

We have demonstrated that myocardial 18F-fluoride PET uptake is increased in patients with TTR amyloid, with TBR values providing quantification and clear discrimination from other similar conditions such as AL amyloid and aortic stenosis. This hybrid imaging technique may, therefore, help in the diagnosis of ATTR amyloid. 
A

\section{Septal uptake}

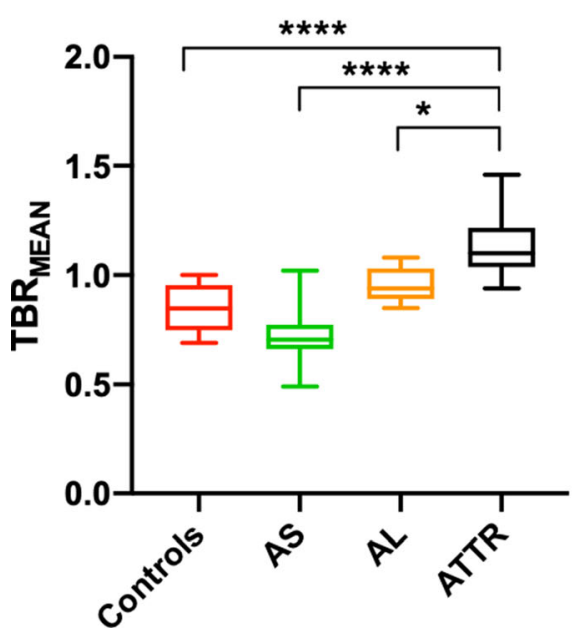

B

\section{LGE uptake}

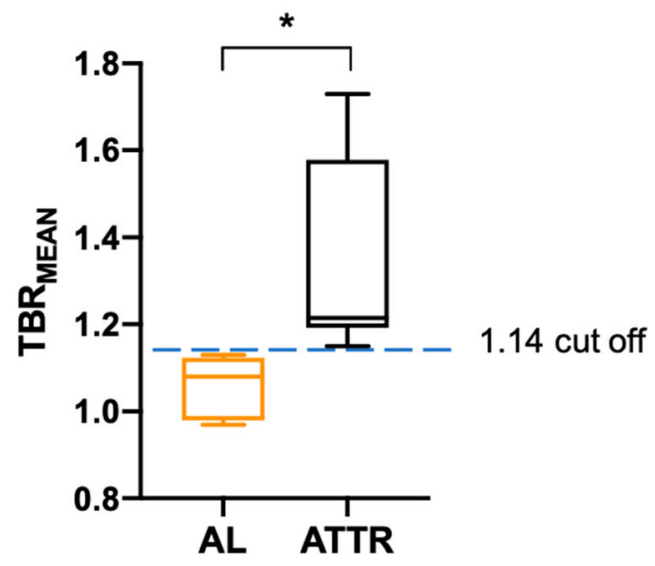

Figure 3. Comparison of myocardial $\mathrm{TBR}_{\mathrm{MEAN}}$ across all cohorts and both subtypes of cardiac amyloid. TBR MEAN $_{\text {in }}$ graph A allows differentiation between ATTR and all cohorts. Further differentiation between amyloid subtypes can be appreciated in graph B with TTR displaying greater uptake than AL. Moreover, within areas of LGE, a cut-off value of $>1.14$ gives $100 \%$ sensitivity and $100 \%$ specificity to detect ATTR over AL. Ordinary one-way ANOVA and unpaired $t$ test. $*<0.05, * *<0.01, * * *<0.001, * * * *<0.0001$.

\section{NEW KNOWLEDGE GAINED}

- We identified that ATTR binds 18F-fluoride more avidly than AL and the negative control groups

- We present 18 F-fluoride PET/MR as a hybrid imaging technique able to distinguish between amyloid subtypes, phenotypically similar patients with aortic stenosis and age/sex-matched controls.

- This may improve our ability as clinicians to make the correct diagnosis and offer appropriate disease specific treatment.

\section{Acknowledgements}

We acknowledge the support of Siemens Healthineers in the use of the Radial Selfgating MR Works-In-Progress package. This sequence is based on contributions from Simon Bauer, Robert Grimm, and Matthias Fenchel. The authors would also like to acknowledge the team of Radiographers and PET Physicists within Queens Medical Research Institute Imaging Facility with special thanks to $\mathrm{Mr}$ David Brian, Mr Ken Dolan, and Dr Tim Clark.

\section{Disclosures}

None of the authors disclose any competing interests.

\section{Open Access}

This article is licensed under a Creative Commons Attribution 4.0 International License, which permits use, sharing, adaptation, distribution and reproduction in any medium or format, as long as you give appropriate credit to the original author(s) and the source, provide a link to the Creative Commons licence, and indicate if changes were made. The images or other third party material in this article are included in the article's Creative Commons licence, unless indicated otherwise in a credit line to the material. If material is not included in the article's Creative Commons licence and your intended use is not permitted by statutory regulation or exceeds the permitted use, you will need to obtain permission directly from the copyright holder. To view a copy of this licence, visit http://creativecommons.org/licenses/by/4.0/.

\section{Author Contributions}

$M R D, D E N, M G T, P M R$, and ZAF conceived and planned the experiments. NS, RE, MGT, MRD, TP, CL, EvB, and AM carried out the experiments. JPMA, MRD, MGT, and DEN contributed to the interpretation of the results. JPMA took the lead in writing the manuscript with MRD. All authors provided critical feedback and helped shape the research, analysis, and manuscript. 


\section{References}

1. Merlini G, Bellotti V. Molecular mechanisms of amyloidosis. N Engl J Med 2003;349:583-96.

2. Ruberg FL, Berk JL. Transthyretin (TTR) cardiac amyloidosis. Circulation 2012;126:1286-300.

3. Skinner M, Sanchorawala V, Seldin DC, et al. High-dose melphalan and autologous stem-cell transplantation in patients with AL amyloidosis: an 8-year study. Ann Intern Med 2004;140:8593.

4. Maurer MS, Schwartz JH, Gundapaneni B, et al. Tafamidis treatment for patients with transthyretin amyloid cardiomyopathy. N Engl J Med 2018;379:1007-16.

5. Longhi S, Lorenzini M, Gagliardi $\mathrm{C}$, et al. Coexistence of degenerative aortic stenosis and wild-type transthyretin-related cardiac amyloidosis. JACC Cardiovasc Imaging 2016;9:325-7.

6. Treibel TA, Fontana M, Gilbertson JA, et al. Occult transthyretin cardiac amyloid in severe calcific aortic stenosis: Prevalence and prognosis in patients undergoing surgical aortic valve replacement. Circ Cardiovasc Imaging 2016;9:e005066.

7. Nietlispach F, Webb JG, Ye J, et al. Pathology of transcatheter valve therapy. JACC Cardiovasc Interv 2012;5:582-90.

8. Dweck MR, Joshi S, Murigu T, et al. Midwall fibrosis is an independent predictor of mortality in patients with aortic stenosis. J Am Coll Cardiol 2011;58:1271-9.

9. Azevedo CF, Nigri M, Higuchi ML, et al. Prognostic significance of myocardial fibrosis quantification by histopathology and magnetic resonance imaging in patients with severe aortic valve disease. J Am Coll Cardiol 2010;56:278-87.

10. Barone-Rochette G, Piérard S, De Ravenstein CD, et al. Prognostic significance of LGE by CMR in aortic stenosis patients undergoing valve replacement. J Am Coll Cardiol 2014;64:144-54.

11. Chin CWL, Everett RJ, Kwiecinski J, et al. Myocardial fibrosis and cardiac decompensation in aortic stenosis. JACC Cardiovasc Imaging 2017;10:1320-33.

12. Rapezzi C, Quarta CC, Guidalotti PL, et al. Role of (99m)Tc-DPD scintigraphy in diagnosis and prognosis of hereditary transthyretinrelated cardiac amyloidosis. JACC Cardiovasc Imaging 2011;4:659-70.

13. Gillmore JD, Maurer MS, Falk RH, et al. Nonbiopsy diagnosis of cardiac transthyretin amyloidosis. Circulation 2016;133:2404-12.

14. Blau M, Nagler W, Bender MA. Fluorine-18: A new isotope for bone scanning. J Nucl Med 1962;3:332-4.

15. Blau M, Ganatra R, Bender MA. 18 F-fluoride for bone imaging. Semin Nucl Med 1972;2:31-7.

16. Morgenstern R, Yeh R, Castano A, Maurer MS, Bokhari S. Fluorine sodium fluoride positron emission tomography, a potential biomarker of transthyretin cardiac amyloidosis. J Nucl Cardiol 2018;25:1559-67.

17. Trivieri MG, Dweck MR, Abgral R, et al. F-Sodium Fluoride PET/ MR for the assessment of cardiac amyloidosis. J Am Coll Cardiol 2016;68:2712-4.
18. Robson PM, Dweck MR, Trivieri MG, et al. Coronary artery PET/ MR imaging: Feasibility, limitations, and solutions. JACC Cardiovasc Imaging 2017;10:1103-12.

19. Kolbitsch C, Neji R, Fenchel M, et al. Joint cardiac and respiratory motion estimation for motion-corrected cardiac PET-MR. Phys Med Biol 2018;64:015007.

20. Pawade TA, Cartlidge TR, Jenkins WS, et al. Optimization and reproducibility of aortic valve $18 \mathrm{~F}$-fluoride positron emission tomography in patients with aortic stenosis. Circ Cardiovasc Imaging 2016;9:e005131.

21. Martinez-Naharro A, Treibel TA, Abdel-Gadir A, et al. Magnetic resonance in transthyretin cardiac amyloidosis. J Am Coll Cardiol 2017;70:466-77.

22. Jenkins WS, Vesey AT, Shah AS, et al. Valvular (18)F-fluoride and (18)F-fluorodeoxyglucose uptake predict disease progression and clinical outcome in patients with aortic stenosis. J Am Coll Cardiol 2015;66:1200-1.

23. Abulizi M, Sifaoui I, Wuliya-Gariepy M et al. F-sodium fluoride PET/MRI myocardial imaging in patients with suspected cardiac amyloidosis. J Nucl Cardiol 2019.

24. Martineau P, Finnerty V, Giraldeau G, Authier S, Harel F, Pelletier-Galarneau M. Examining the sensitivity of $18 \mathrm{~F}-\mathrm{NaF}$ PET for the imaging of cardiac amyloidosis. J Nucl Cardiol 2019.

25. Lilleness B, Doros G, Ruberg FL, Sanchorawala V. Establishment of brain natriuretic peptide-based criteria for evaluating cardiac response to treatment in light chain (AL) amyloidosis. Br J Haematol 2019.

26. Lilleness B, Ruberg FL, Mussinelli R, Doros G, Sanchorawala V. Development and validation of a survival staging system incorporating BNP in patients with light chain amyloidosis. Blood 2019;133:215-23.

27. Castaño A, Narotsky DL, Hamid N, et al. Unveiling transthyretin cardiac amyloidosis and its predictors among elderly patients with severe aortic stenosis undergoing transcatheter aortic valve replacement. Eur Heart J 2017;38:2879-87.

28. Dweck MR, Chow MW, Joshi NV, et al. Coronary arterial 18Fsodium fluoride uptake: A novel marker of plaque biology. J Am Coll Cardiol 2012;59:1539-48.

29. Joshi NV, Vesey AT, Williams MC, et al. 18F-fluoride positron emission tomography for identification of ruptured and high-risk coronary atherosclerotic plaques: A prospective clinical trial. Lancet 2014;383:705-13.

30. Irkle A, Vesey AT, Lewis DY, et al. Identifying active vascular microcalcification by (18)F-sodium fluoride positron emission tomography. Nat Commun 2015;6:7495.

31. Moss AJ, Doris MK, Andrews JPM, et al. Molecular coronary plaque imaging using. Circ Cardiovasc Imaging 2019;12:e008574.

Publisher's Note Springer Nature remains neutral with regard to jurisdictional claims in published maps and institutional affiliations. 